\title{
Avaliação comparativa de diferentes fatores desencadeantes de estresse em mulheres docentes de medicina em decorrência da nova rotina de trabalho pelo COVID-19
}

Comparative evaluation of different understanding stress factors in medicine teaching women due

to the new work routine by COVID-19

Evaluación comparativa de diferentes factores desencadenantes del estrés en profesoras de medicina como resultado de la nueva rutina laboral de COVID-19 Centro Universitário São Lucas, Brasil E-mail: ennely@gmail.com

Suelem Damaris David da Silva ORCID: https://orcid.org/0000-0001-5239-0088 Centro Universitário São Lucas, Brasil

E-mail: suelem_dds@hotmail.com

Laura Rayssa Cavalcanti Santos ORCID: https://orcid.org/0000-0001-5590-8399 Centro Universitário São Lucas, Brasil

E-mail: laura.biomed@outlook.com

Alexandre Humberto Florencio Silva ORCID: https://orcid.org/0000-0003-2881-0913 Centro Universitário São Lucas, Brasil

E-mail: alexandrehumberto.edu_@hotmail.com

Gustavo Rodrigues Lopes

ORCID: https://orcid.org/0000-0002-9337-4188 Centro Universitário São Lucas, Brasil

E-mail: Gustavorodrigues_Lopes@hotmail.com

Gabrielle Ohana de Sousa Paixão ORCID: https://orcid.org/0000-0003-1525-329X Centro Universitário Aparício Carvalho, Brasil E-mail: Gabrielle.ohana@icloud.com

Maikson Gustavo Soares de Oliveira ORCID: https://orcid.org/0000-0002-2004-1913 Universidade Estadual da Bahia, Brasil E-mail: profgustaeduca@ gmail.com

Sandro de Vargas Schons

ORCID: https://orcid.org/0000-0001-9811-5356

Universidade Federal de Rondônia, Brasil E-mail: sandroschons@unir.br Igor Mansur Muniz

ORCID: https://orcid.org/0000-0003-0863-6647

Universidade Federal de Rondônia, Brasil E-mail: igor.mansur@unir.br

Fernando Andrade Souza

ORCID: https://orcid.org/0000-0002-9474-9404 Universidade Federal do Paraná, Brasil E-mail: femedvet@yahoo.com.br 


\begin{abstract}
Resumo
A pandemia gerada pela COVID-19 fez com que a população renovasse e mudasse hábitos, utilizando-se de métodos preventivos, como o isolamento social, e distanciamento social. Sendo necessário medidas como a adoção de home office, o que afetou diretamente as docentes do curso de medicina, ao terem que se adaptar as jornadas duplas e triplas de trabalho, com a execução da docência a partir do seu lar. Com isso objetivou-se avaliar os fatores desencadeantes de estresse nessa nova modalidade de trabalho, quando comparado a jornada anterior. A pesquisa foi realizada utilizandose da aplicação de questionários por plataforma online com questões de múltiplas escolhas, contendo critérios de inclusão e exclusão. Dentre as entrevistadas 55\% se demostraram estressadas, estando 40\% delas pouco satisfeitas e $35 \%$ insatisfeita com a modalidade de home office, esse alto nível de estresse segundo Cabral (2007) é o principal gerador de ansiedade culminando para o desencadeamento de outras alterações comportamentais. Concluindo-se assim que a aplicação de questionário foi efetivo para esse tipo de análise, como também sendo notado fatores estressantes causados por essa nova modalidade de trabalho.
\end{abstract}

Palavras-chave: Pandemia; Professoras; Médicas.

\begin{abstract}
The pandemic generated by COVID-19 caused the population to renew and change habits, using preventive methods, such as social isolation, and social distance. It is necessary to take measures such as the adoption of a home office, which directly affected the teachers of the medical course, as they had to adapt the double and triple working hours, with the execution of teaching from their home. Thus, the objective was to evaluate the factors that trigger stress in this new type of work, when compared to the previous day. The research was carried out using the application of questionnaires by online platform with multiple choice questions, containing inclusion and exclusion criteria. Among the interviewees, $55 \%$ were stressed, with $40 \%$ of them not very satisfied and $35 \%$ dissatisfied with the home office modality, this high level of stress according to Cabral (2007) is the main generator of anxiety culminating in the triggering of other behavioral changes. Thus concluding that the application of a questionnaire was effective 2 for this type of analysis, as well as being noticed stressful factors caused by this new modality of work.
\end{abstract}

Keywords: Pandemic; Teachers; Doctors.

\title{
Resumen
}

La pandemia generada por COVID-19 provocó que la población renovara y cambiara hábitos, utilizando métodos preventivos, como el aislamiento social y el distanciamiento social. Fueron necesarias medidas como la adopción de una oficina en casa, que afectó directamente a los profesores de la carrera de medicina, ya que debían adaptarse a los turnos de trabajo dobles y triples, con la docencia desde su casa. Con ello, el objetivo fue evaluar los factores desencadenantes del estrés en esta nueva modalidad de trabajo, en comparación con la jornada anterior. La investigación se realizó mediante cuestionarios a través de una plataforma en línea con preguntas de opción múltiple, que contienen criterios de inclusión y exclusión. Entre los entrevistados, el 55\% estaba estresado, el $40 \%$ poco satisfecho y el $35 \%$ insatisfecho con la modalidad home office, este alto nivel de estrés, según Cabral (2007), es el principal generador de ansiedad, culminando en el desencadenante de otros cambios de comportamiento. Así, se concluye que la aplicación de un cuestionario resultó eficaz para este tipo de análisis, además de los factores estresantes provocados por este nuevo tipo de trabajo.

Palabras clave: Pandemia; Profesores; Doctores.

\section{Introdução}

Dentre os sintomas causados pela COVID-19, por ser tratar de uma patologia de alterações multissistêmica, pode se evidenciar as geradas por estresse e danos psicológicos, potencializados pelo isolamento social como também a nova configuração do estilo de vida, com o trabalho desenvolvido em ambiente domiciliar (López et al. 2020).

As consequências que a pandemia pelo COVID-19 tem gerado na saúde mental, tem estimulado inúmeras pesquisas, porém pouco se tem de resultados até então, por se tratar de um evento recente, entretanto com base em pesquisas anteriores realizadas em outros surtos infecciosos, causadores também de alterações mentais e psicológicas, pode-se observar na população o agravamento destes com o passar do tempo (Schmidt, 2020).

Como medida drástica para o controle da pandemia foram adotadas inúmeras medidas, dentre elas a de adoção repentina ao home office, por uma adaptação forçada a essa nova realidade, os lares foram obrigados a se tornarem um apêndice das empresas, como também dos estabelecimentos de ensino, desconfigurando as principais estruturações das entidades dentro da sociedade (Savic, 2020; Waizenegger; McKenna; Bendz, 2020). 
Os professores tanto do ensino público, como do privado nos mais diversos níveis de formação e ensino, passaram a realizar tarefas de forma inesperada, utilizando-se de plataformas digitais e aparatos tecnológicos, sem mesmo terem recebido um preparo para tais, tendo que em muitos casos recorrer ao improviso para a execução do trabalho, como também para a simulação de situações que o fizesse ser compreendido, utilizando assim do seu ambiente doméstico, expondo-o e até mesmo gerando constrangimentos, dividindo-se ainda entre as atividades domesticas e familiares (Souza, et al. 2021).

Um conjunto de pressões que ocorrem do conflito trabalho-família, surgem com a necessidade em desempenhar simultaneamente os papeis profissionais e pessoais, sendo agravado ainda por isso ocorrer em um único ambiente, o que dificulta a execução de um deles ou de ambos (Zhao, et al. 2020). Há relatos por alguns pais de que toda essa mudança atuou de forma positiva, por permitirem uma maior aproximação e dedicação de tempo aos filhos, mas não descartam a afirmação da dificuldade em conciliar papeis diferentes dentro do ambiente domiciliar (Pluut, et al. 2018).

Dentro dessa problemática objetivou-se a realização de um estudo, através da aplicação de questionários, em que foram pontuados os aspectos geradores de estresses em mulheres docentes do curso de medicina, comparando assim o atual período com a configuração anterior da rotina de trabalho.

\section{Materiais e Métodos}

Essa pesquisa foi submetida ao Comitê de Ética em Pesquisa Humana - CEP e aprovado sob número e parecer 4.581.683. A pesquisa foi realizada com docentes do curso de medicina de uma instituição de ensino privado na cidade de Porto Velho-RO, nesta pesquisa foram avaliados questionários respondidos por 20 participantes que atenderam aos critérios de inclusão e exclusão.

A pesquisa foi feita por meio da plataforma "Google Forms", fornecendo assim os dados que foram coletados de acordo com as respostas das entrevistadas. A abordagem foi feita diretamente às docentes por meio das plataformas de redes sociais, sendo enviado então um link para que elas pudessem estar respondendo.

Critérios de inclusão: Ser docente de medicina do Centro Universitário São Lucas, do sexo feminino e assinar o Termo de Consentimento Livre e Esclarecido (TCLE).

Critérios de exclusão: Não concordar com os termos da participação da pesquisa e/ou não assinar o Termo de Consentimento Livre e Esclarecido (TCLE).

A pesquisa foi realizada com o auxílio de um questionário dividido em duas grandes partes, sendo a primeira de identificação e caracterização da docente, e uma segunda voltada para à avaliação proposta, elaborado com questões de múltipla escolha, possibilitando assim avaliar o nível de estresse de forma quantitativa e qualitativa com base na jornada de trabalho atual comparando com a anterior por ela exercida. O processamento dos dados foi realizado de forma computadorizada, com formatação de tabelas e gráficos, no programa Microsoft Office Excel 2007.

\section{Resultados}

O formulário da pesquisa foi respondido por 20 docentes do curso de medicina de uma instituição de ensino superior, sendo que todas aceitaram e concordaram com o Termo de Consentimento Livre e Esclarecido (TCLE). Das entrevistadas 35\% (7/20) são solteiras, casadas 45\% (9/20), divorciadas 15\% (3/20) e outras 5\% (1/20). A cor de autodeclaração apresentada foi de $55 \%$ (11/20) para cor parda e 45\% (9/20) para a branca. Em se tratando do credo religioso, verificou-se 45\% (9/20) evangélicas, $35 \%$ (9/20) católicas e na opção outras $20 \%$ (4/20).

A renda se demonstrou por elas para o cumprimento das necessidades básicas (educação, saúde, moradia e entretenimento) para 15\% (3/20) excelente, 25\% (5/20) muito satisfatória, 55\% (11/20) satisfatória e 5\% (1/20) ruim. 
O nível de titulação dentre as pesquisadas, apresentou-se com apenas graduação 15\% (3/20), especialização 75\% (15/20), mestra 5\% (1/20) e doutora 5\% (1/20). Para o tempo de formação em anos apenas 15\% (3/20) tem de 2 a 4, 45\% (9/20) com 4 a $8,35 \%(7 / 20)$ com 8 a 15 e $5 \%(1 / 20)$ com mais de 15.

Sendo a segunda parte do questionário direcionada para a avaliação do perfil de estresse das docentes, quando comparado a rotina de trabalho pré e durante a pandemia da COVID-19, gerado pela nova modalidade de realização de trabalho no lar.

O número de filhos apresentado por elas demonstrou-se de 5\% (1/20) tendo 3 filhos, 35\% (7/20) tendo dois filhos, $15 \%$ (3/20) apenas 1 filho e 45\% (9/20) não tendo. Em relação aos episódios de estresse, relacionado a nova jornada e modalidade de trabalho durante a pandemia, demonstrou estar presente na rotina de forma extremamente frequente em 20\% (4/20), muito frequente $50 \%$ (10/20), frequente $20 \%$ (4/20) e não tem gerado estresse em $10 \%(2 / 20)$.

Foram apresentados dados em relação ao auxílio de funcionários durante a pandemia, como sendo-o por empregada doméstica em 60\% (12/20), babá em 20\% (4/20), diarista 5\% (1/20) e outros 15\% (3/20).

A capacidade de conciliar a jornada de trabalho com os afazeres pessoais, foi categorizada em uma escala de 0 a 5 , em que zero é menor eficácia e cinco em uma maior eficácia, atribuiu-se em 10\% (2/20) para nota 5, 15\% (3/20) para nota 4, 60\% $(12 / 60)$ para nota $3,5 \%(1 / 20)$ para nota 2 e $10 \%(1 / 20)$ para nota 1.

$\mathrm{Na}$ jornada média de carga horaria semanal de trabalho durante a pandemia, foi verificada as porcentagens de $65 \%$ (13/20) maior que 40 horas, $15 \%$ (3/20) de 20 a 40 horas, 10\% (2/20) de 15 a 20 horas como também o mesmo para de 8 a 15 horas.

A disponibilidade em estar realizando atividades recreativas com a intenção de melhoria na qualidade de vida e diminuição do estresse, foi atribuído em uma escala de 0 a 5 , em que zero é menor eficácia e cinco uma maior, como demonstrado no Gráfico 1.

Gráfico 1 - Classificação de disponibilidade de estar realizando atividades recreativas, com a intenção de melhoria na qualidade de vida e diminuição do estresse, pelas docentes durante a pandemia por COVID-19.

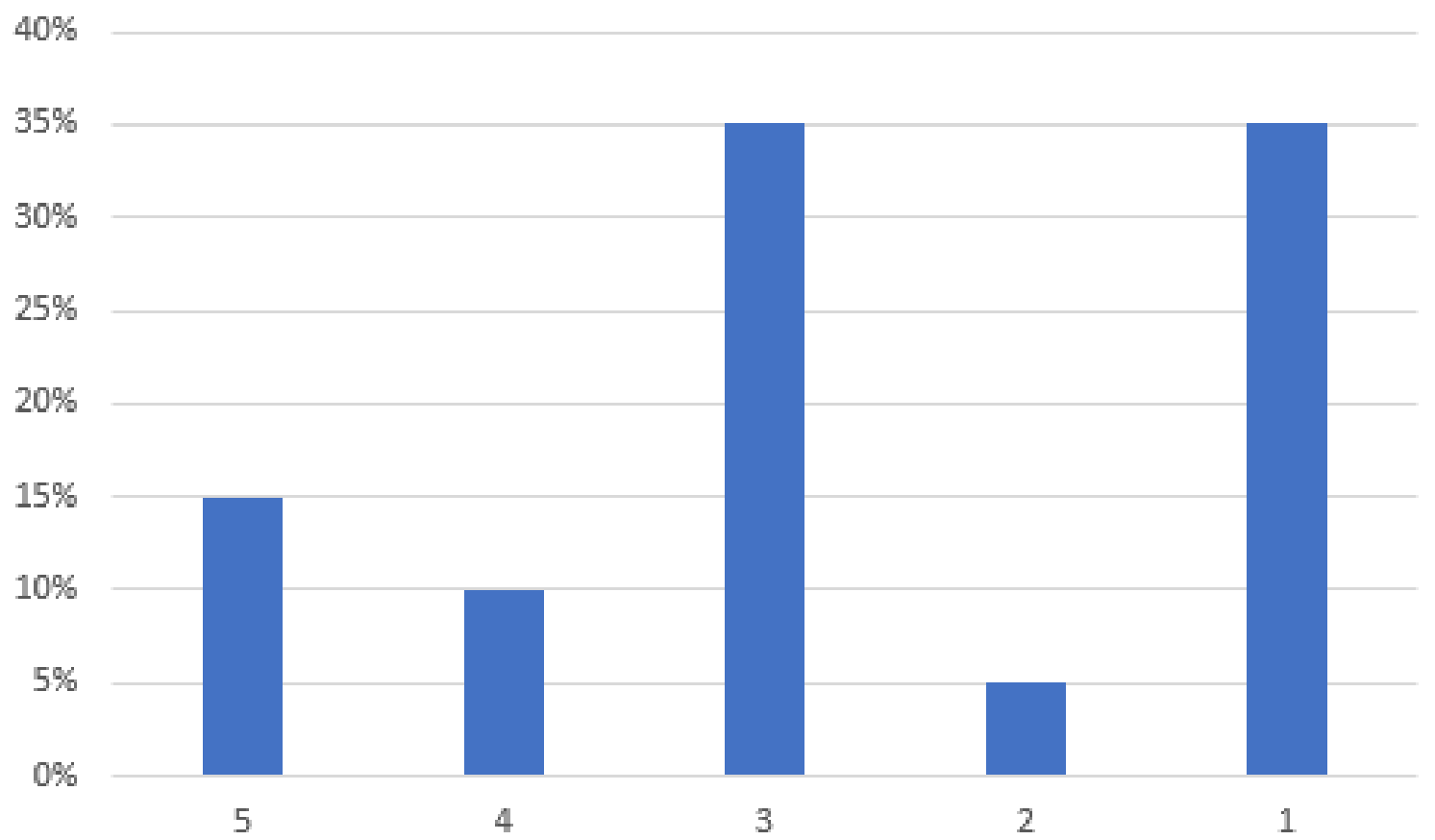

Fonte: Elaborado a partir de pesquisa conduzida. 
A prática de exercícios físicos foi verificada pela frequência em que esses estavam sendo praticados por elas, durante o período de pandemia, estando 50\% (10/20) sem realizar nenhuma atividade física, 30\% (6/20) praticando 3 vezes por semana, $15 \%(3 / 20) 4$ vezes por semana e 5\% (1/20) duas vezes na semana.

Em relação a condição emocional no momento da pesquisa quando comparado a rotina de trabalho antes da pandemia, e ao período de pandemia por COVID-19, foi solicitado então uma classificação do estado emocional, sendo apresentadas então as seguintes porcentagens (Gráfico 2).

Gráfico 2 - Classificação do estado emocional das participantes, apresentado em porcentagem, quando comparado a rotina de trabalho antes da pandemia, e ao período de pandemia por COVID-19.

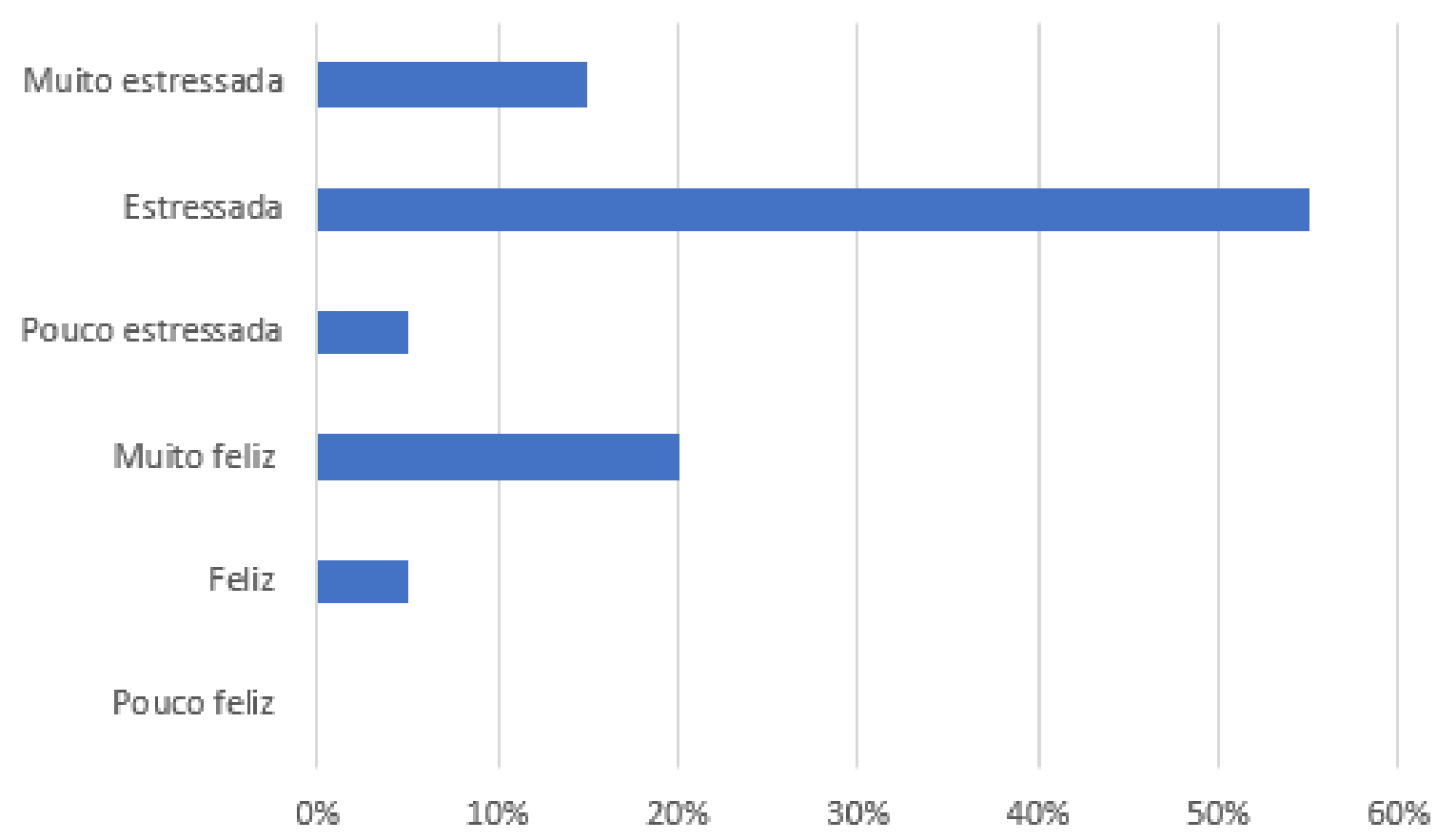

Fonte: Elaborado a partir de pesquisa conduzida.

Na percepção das médicas docentes em relação ao seu amparo em momentos de estresse durante a pandemia da COVID19, relacionado ao vínculo amparador, tendo como respostas; não tendo amparo, amparada por amigos, pela religião, por psicólogos/psiquiatras, por animais de estimação e por familiares, apenas as últimas duas opções foram indicadas por elas como fontes amparadoras durante esse período, apresentando assim respectivamente 15\% (3/20) e 85\% (17/20). A busca pelo apoio de profissionais psicólogos durante esse período foi verificada, em que 50\% (10/20) responderam que sim e as outras que não.

Tratando-se de alterações comportamentais ligadas a nova jornada de trabalho, foram verificadas a manifestação das principais delas pelas docentes, apresentado então no questionário; ansiedade marcada por 40\% (8/20), depressão por 10\% (2/20), síndrome do pânico em 10\% (2/20) e as que disseram não terem desenvolvido nenhuma dessas alterações em 40\% (8/20).

Os efeitos da pandemia na modalidade de docência por home office no curso de medicina, e a nova jornada de trabalho com conciliação dos afazeres pessoais, trouxe diferentes modalidades de satisfação, apresentaram-se como; pouco satisfeita em $40 \%$ (8/20), muito satisfeita 10\% (2/20), imensamente satisfeita 15\% (3/20) e insatisfeita 35\% (7/20).

\section{Discussão}

Segundo Crespo e Gurovitz (2002) definiram a "capacidade" como sendo o poder de manutenção das necessidades básicas do indivíduo, estando ela atrelada a renda, sendo que quanto maior então forem os rendimentos melhor será a capacidade 
da pessoa em manter as suas necessidades básicas, vindo de encontro ao manifestado nessa pesquisa pelas entrevistadas, com 95\% delas nos níveis de: excelente, muito satisfatório e satisfatório contra apenas 5\% de ruim, para o critério de satisfação com a renda.

Godinho (2011) descreve as necessidades básicas humanas como sendo as físicas (alimentação, vestuário e mobiliário) e as sociais (saneamento, saúde, trabalho, educação e cultura), sendo que o suprimento delas geram o bem-estar. É sabido que na maioria da população, a disponibilidade de recursos oriundas da "capacidade", está ligado a formação acadêmica e obtenção de títulos.

O nível de estresse quando comparado entre os gêneros, pode ser facilmente observado com maior relevância nas mulheres, 55\% das docentes manifestaram terem mais de um filho, podendo ser um desencadeador de estresse, associado aos cuidados dispensados a eles, como também a dupla jornada de trabalho, sendo agravado pela mudança brusca na docência para a modalidade home office, sem uma preparação previa para tal, por medidas emergenciais (Areias \& Guimarães, 2004).

Uma das formas de diminuir a sobrecarga materna, associada à dupla ou tripla jornada de trabalho, está em ter a ajuda de auxiliares prestadores de serviços, podendo destacar-se dentre os resultados a porcentagem de $60 \%$ das entrevistadas, em lançarem mão da utilização dos serviços de empregada doméstica, sendo esse o binômio necessidade - prestação de serviço, a fim de auxiliar e diminuir o estresse gerado pelos afazeres cotidianos do lar (Oliveira \& Andrade, 2020).

As empregadas domésticas, as cuidadoras, os trabalhadores informais, os jovens e os pretos foram de uma forma geral os que mais tiveram redução da jornada de trabalho e despontam nas listas de desempregados, em contra partida, os trabalhadores com maiores níveis de escolaridade, principalmente dentre os da área da saúde, podem ser os citados com aumento de jornada de trabalho e sobrecarga, vindo ao encontro do demonstrado pelas entrevistadas, que são mulheres, médicas e docentes no nível superior, apresentando uma jornada semanal de trabalho maior que 40 horas, para 65\% das delas (Barbosa; Hecksher; Costa, 2020). Portanto, com o aumento da carga de trabalho realizado pelas docentes médicas durante a pandemia, reduziu-se por demais o tempo livre para o exercício físico e atividades recreativas, sendo que $50 \%$ delas conseguiram manter a frequência de atividades em no mínimo 3 vezes na semana ou mais. Segundo Ribeiro et al., (2020), é de fundamental importância a prática do lazer e atividades físicas, sendo essa uma necessidade humana caracterizada pela vivência lúdica de manifestações culturais no tempo/espaço social.

Quanto maior for a disponibilidade de tempo para a realização de atividades recreativas, exercícios físicos e até mesmo um tempo para o ócio, refletirá então de forma significativa na qualidade de vida. Quando perguntado em relação ao estado emocional, $55 \%$ das docentes estudadas manifestaram a condição de estressada devido aos efeitos dessa nova rotina de trabalho e a baixa disponibilidade para realização dessas atividades (Batista; Ribeiro; Junior, 2012; Marcelino, 2012).

Os altos níveis de estresse fazem com que venha a ser requerido o acolhimento por outros indivíduos, por sermos seres socias. A principal busca por acolhimento foi ao seio familiar, relatado por $85 \%$ delas. A família por natural funciona como esse local de proteção desde a infância e permanecendo por toda a vida (Nuñez, 2008; Brasil, 2020).

Ao analisar os dados da pesquisa, evidenciou-se também a busca por amparo emocional, nos animais domésticos, visto que $15 \%$ buscam o amparo neles. Segundo o autor Albuquerque (2021) e o que já havia sido relatado em 2013 pelo IBGE, estima-se que há mais animais de estimação nas casas do que crianças, demonstrando como a convivência com os animais domésticos é fundamental para o equilíbrio emocional.

Dentre as alterações comportamentais mais comum podemos destacar o surgimento de ansiedade em $40 \%$ delas, devido ao estresse gerado, o que levou a uma alta demanda energética, devido a uma grande atividade a nível de sistema neuromuscular e nos demais tecidos, sendo essa a primeira manifestação a surgir após os picos de estresse, podendo ainda evoluir para outras alterações comportamentais (Cabral, et al., 2007). 
Uma pesquisa realizada com profissionais que passaram a trabalhar em home office, apresentou 27\% dos entrevistados com os rendimentos severamente comprometidos, desentoando dos valores encontrados nesta, em que $40 \%$ delas 9 demonstraram insatisfação total com essa modalidade, já outros 9\% declararam que tiveram o potencial de trabalho otimizado, compatível com os $10 \%$ de médicas docentes, que demonstraram muita satisfação com esse novo método (Magnan, et al. 2020).

\section{Conclusão}

Conclui-se assim que o método de questionário é eficiente para avaliação da percepção de efeitos geradores de estresse em docentes do curso de medicina, como também capaz de identificar fatores desencadeantes de estresse, quando comparado à rotina de médicas docentes no período anterior ao de durante a pandemia por COVID-19.

\section{Referências}

Albuquerque, N. S. et al. (2020). Cães e gatos domésticos em tempos da pandemia da COVID-19. ReseachGate. Technical Report.

Areias, M. E. Q. \& Guimaraes, L. A. M. (2004). Gênero e estresse em trabalhadores de uma universidade pública do estado de São Paulo. Psicologia em Estudo, 9(2): 255-262.

Barbosa, A. L. N. H., Hecksher, M. \& Costa, J. S. C. (2020). Mercado De Trabalho E Pandemia Da Covid-19: Ampliação De Desigualdades Já Existentes? Mercado de Trabalho. Notas Técnicas. DOI: http://dx.doi.org/10.38116/bmt69/notastecnicas1

Batista, J., Ribeiro, O. C. F. \& Junior, P. N. (2012). Lazer e promoção de saúde: uma aproximação conveniente. Licere, 15(2): 1-16.

Brasil. (2020). O papel da família na promoção da saúde mental/ José Manoel Bertolote... [et al.]; Secretaria Nacional da Família (Coordenação); Ministério da Mulher, da Família e dos Direitos Humanos.

Cabral, A. P. T. et al. (1997). O Estresse e as Doenças Psicossomáticas. Revista De Psicofisiologia, 1(1).

Crespo, A. P. A. \& Gurovitz, E. (2002). A pobreza como um fenômeno multidimensional. RAE-eletrônica, 1(2).

Godinho, I. C. (2011). Pobreza e desigualdade social no Brasil: um desafio para as Políticas Sociais. Eixo Temático: O Serviço Social e o Desenvolvimento. Anais do I Circuito de debates acadêmicos. UFRJ CODE.

Magnan, E. S. et al. (2020). Cartilha sobre Home Office em tempos de pandemia: O que você precisa saber para trabalhar bem e com saúde. Porto Alegre: PUCRS/Vitória: UFES/ PUC-Rio.

Marcellino, N. C. (2012). Estudos do lazer: uma introdução. (5a ed.), Autores Associados.

Nuñez, B. (2008). Família y discapacidad: de la vida cotidiana a la teoria. Lugar Editorial.

López, V. L. D. et al. (2020). Estrés en docentes universitarios: promoviendo el bienestar en tiempos de coronavirus. Dissertação - Universidad Cooperativa de Colombia Faculta de psicologia.

Oliveira, A. P. \& Andrade, M. L. E. S. (2020). Trabalho Doméstico E Pandemia: o meio ambiente laboral e a possibilidade de acidente do trabalho. Revista Cientifica Faculdade Unimed. 2(1).

Pluut, H. et al. (2018). Social support at work and ate home: Dual buffering effect in the work-family conflict process. Organizational Behavior and Human Decision Processes, 146: 1-13.

Ribeiro, O. C. F. et al. (2020). Os impactos da pandemia da COVID-19 no lazer de adultos e idosos. Revista do Programa de Pós-graduação Interdisciplinar em Estudos do Lazer - UFMG. 23(3).

Savic, D. (2020). COVID 19 and work from home: Digital transformation of the workforce. The Grey Journal. 16(2): 101-104.

Schmidt, B. (2020). Saúde mental e intervenções psicológicas diante da pandemia do novo coronavírus (COVID-19). Estudos de Psicologia, 37.

Souza, K. R. et al. (2021). Trabalho remoto, saúde docente e greve virtual em cenário de pandemia. Trabalho, Educação e Saúde, 19.

Zhao, Y. et al. (2020). Parent's shift work in connection with work-family conflict and mental health: examining the pathways for mothers and fathers. Journal of Family Issues, 1-29.

Waizenegger, L. et al. (2020). An affordance perspective of team collaboration and enforced working from home during COVID 19. European Journal of Information Systems, 29(4), 429-442. 